\title{
Electrodeposition and characterisation of Nilgelatin microgels coatings
}

Aaron Gibb ${ }^{\mathrm{a}}$, Andrejs Vorobjovs ${ }^{\mathrm{a}}$, Zofia Jagoda ${ }^{\mathrm{b}}$, Juliusz Winiarski ${ }^{\mathrm{c}}$, Alicja Stankiewicz ${ }^{\mathrm{a}^{*}}$

${ }^{a}$ Edinburgh Napier University, School of Engineering and the Built Environment, 10 Colinton Road, EH10 5DT, Edinburgh, United Kingdom

${ }^{b}$ Wroclaw University of Economics, Department of Inorganic Chemistry, Komandorska 118/120, 53-345 Wroclaw, Poland

${ }^{c}$ Wroclaw University of Science and Technology, Faculty of Chemistry, Wybrzeze Wyspianskiego 27, 50-370 Wroclaw, Poland

*corresponding author; a.stankiewicz@ napier.ac.uk, Edinburgh Napier University, School of Engineering and the Built Environment, 10 Colinton Road, EH10 5DT, Edinburgh, United Kingdom

Mr Aaron Gibb - MSc student at Edinburgh Napier University involved in research on electrodeposition of Nilgelatin microgels coatings.

Mr Andrejs Vorobjovs - Beng student at Edinburgh Napier University involved in research on mechanical properties of Nilgelatin microgels coatings.

Ms Zofia Jagoda - PhD student at Wroclaw University of Economics in Poland. She works on preparation of various types of microgels, as well as entrapment and release of corrosion inhibitors.

Dr Juliusz Winiarski - Assistant Professor at Wroclaw University of Science and Technology in Poland. His research includes but is not limited to manufacture and characterisation of nickel coatings electrodeposited from eutectic solvents, to study the catalytic activity of perovskites, to study corrosion mechanism of protective coatings. He frequently publishes results of his work in international journals.

Dr Alicja Stankiewicz - Researcher and Lecturer at Edinburgh Napier University with an expertise in the fields of electroless and electrodeposition of protective coatings, as well as corrosion science. She is supervising $\mathrm{PhD}, \mathrm{MSc}$, Honours projects and involves her students in her research. She is a Chartered Scientist and a Member of the Institute of Materials, Minerals and Mining. 


\section{Electrodeposition and characterisation of Nilgelatin microgels coatings}

This work focuses on implementing microgels for carrying self-healing substances in protective coatings. Nilgelatin microgels coatings were manufactured using electroplating techniques. Microscopic analysis was carried out to establish the presence of microgels in the coating. This was followed by an investigation into the influence of the microgels on the mechanical properties and corrosion resistance of the coatings. The composite Nilgelatin microgels coating exhibited better wear resistance and higher hardness than the plain nickel coating. Electrochemical impedance analysis showed the difference in the behaviour of the coatings in $3.5 \mathrm{wt} . \% \mathrm{NaCl}$ solution. With the time of the immersion, the resistance of the nickel coatings decreased from 28 to $14 \mathrm{k} \Omega \mathrm{cm}^{2}$, while the composite coatings increased 5.8 to $10 \mathrm{k} \Omega \mathrm{cm}^{2}$. At this stage of the research, only microgels without any active compounds have been used, so no information related to the self-healing characteristics of coatings is currently available.

Keywords: nickel composite coating; self-healing; microgel; gelatin; corrosion; electrodeposition

\section{Introduction}

The severe and costly damage caused by corrosion continues to push material scientists and engineers to develop new and more efficient methods for corrosion prevention and protection in all areas of industry such as aerospace, automotive, energy and structural engineering. One of the most effective methods for achieving this has been through the application of protective coatings. The application of such coatings is dependent on the type of coating being applied (organic or inorganic), the operating conditions and the required service life of the material during operation. Even though a high-performance quality coating regarding adhesion, mechanical properties and durability can be achieved, there are various mechanical, chemical and thermal impacts experienced by products during service that result in the formation of micro-cracks that lead to premature failure of coating systems [1]. The maintenance and repair of damaged coatings on a large industrial scale are costly. Because of this, attention and research have been focused on the development of advanced coatings as a way of providing more extended service durability and increased corrosion protection of material surfaces. 
Since the development of smart materials, there has been an increasing demand for materials to exhibit additional multifunctional properties [2]. The ability of such materials to self-repair damage caused by external mechanical or chemical damage is a highly attractive feature. The emergence of smart coatings has led to a new generation of protection systems. Rather than act as just a barrier between the substrate and the environment, smart coatings are designed to respond to the environment and aid in enhancing the coatings life and functionality by selfrepairing defects and cracks.

At present, the primary focus has been on the manufacture of coatings with micro- or nanocapsules containing active substances, like corrosion inhibitors [3-5]. These capsules consist of a solid polymeric shell that house a liquid core. When the coating becomes damaged due to physical, internal or external stresses micro-cracks form and propagate through the coating. This results in rupturing the shell of the capsules and releasing the healing agent, which in turn reacts with the environment to fill and seal the crack obstructing the penetration of water, oxygen or corrosive medium from the materials substrate. However, the synthesis complexity and demand for less environmentally damaging materials has resulted in researchers considering alternative approaches to microcapsules. A new option currently being investigated is the use of micron-sized gel particles (microgels). Although research regarding microgels as a potential carrier for corrosion inhibitors is relatively new, it has shown microgels to be a suitable alternative for providing coating systems with self-healing capabilities [6]. The preparation of the gels is much simpler than capsule synthesis and inhibitor filling as it can be achieved using a single-pot process. Further research supporting this, is the production and co-deposition of gelatin microgels into a nickel-phosphorous coating via electroless plating [7].

In this work, the co-deposition of gelatin microgels into a nickel coating applied by electroplating was investigated. The gelatin microgels could be used as carriers of active substances, like corrosion inhibitors, and could improve the overall performance of nickel 
coatings by the introduction of self-healing properties. Mechanical properties like hardness and wear resistance, as well as corrosion resistance of the composite Nilgelatin microgels coatings, were tested and compared to properties of plain nickel coatings to determine if the presence of the microgels in the coatings does not influence negatively on their characteristics.

\section{Materials and methods}

\section{Substrate pretreatment}

Samples of stainless steel (AISI 306) and copper coupons measuring 25x30x1mm were used as the cathode for the electrodeposition process. Copper samples were used for SEM inspection by providing a clear compositional difference between the coating and the substrate.

A $10 \%$ hydrochloric acid solution was used for the activation and removal of scale and oxide layers presence on both the stainless steel and copper samples before plating. Stainless steel and copper samples were immersed into the acidic solution at an elevated temperature of $55^{\circ} \mathrm{C}$ for 1 minute and 30 seconds, respectively. Samples were then thoroughly rinsed and dipped into de-ionised water. Following the pre-treatment process, samples were immediately electroplated.

\section{Gelatin microgels preparation}

Non-cross-linked gelatin microgels were prepared by modified water-in-oil emulsion method described in [7] $10 \%(\mathrm{w} / \mathrm{v})$ gelatin solution in distilled water was heated to the $40^{\circ} \mathrm{C}$ and added dropwise to the paraffin oil preheated to the same temperature. The oil phase was used with the $1 \%(\mathrm{v} / \mathrm{v})$ content of nonionic surfactant Span80. The resulting mixture was stirred at $1250 \mathrm{rpm}$ for $15 \mathrm{~min}$ to prepare 1:10 (v/v) water-in-oil emulsion and then was cooled in an ice bath to $4^{\circ} \mathrm{C}$. Next, cold acetone, in slight volume excess to the oil phase, was added in order 
to dehydrate and separate microspheres from oil. Stirring at $4{ }^{\circ} \mathrm{C}$ was continued for $1 \mathrm{~h}$. The obtained microgels were filtered off under reduced pressure with glass microfiber filters (Whatman Grade GF/A, $1.6 \mu \mathrm{m}$ pore size) and washed several times with cooled acetone, then air-dried at room temperature. Dry gelatin microgels were thermally cross-linked by heating at $150^{\circ} \mathrm{C}$ for $16 \mathrm{~h}$.

\section{Coatings deposition}

A nickel sulphamate bath was selected as a suitable electrolyte to aid in the co-deposition and production of Nilgelatin microgels composite coatings. The fast deposition rate, lowtemperature capabilities [8], reduced nickel chloride concentration and $\mathrm{pH}$ range of solution offered suitable and adaptable conditions. The composition of the bath and process parameters for electrodeposition of plain nickel coatings are presented in Table 1. The chloride content was kept low to achieve minimum stress, without the use of stress-reducing additives. To obtain composite coatings, $3 \mathrm{~g} \mathrm{dm}^{-3}$ of gelatin microgels were added to the basic bath. The bath was magnetically stirred with rpm of 200.

The $\mathrm{pH}$ value was selected in reference to optimal conditions of electrodeposition of nickel from the sulphamate bath to reduce stresses within the deposit [9] and to aid the gelatin particles with a positive charge [10], which promotes their movement towards the cathode.

Plain nickel and composite coatings had a thickness of around $10 \mu \mathrm{m}$.

\section{Zeta potential and particles size measurement}

The zeta potential of gelatin microgels was determined with a Malvern Zetasizer-nano in deionised water across a range of $\mathrm{pHs}$ including $4,5,7$ and 10 . The $\mathrm{pH}$ was adjusted using $\mathrm{HCl}$ or $\mathrm{NaOH}$. A 10-micron filter was used to ensure only particles were dispersed within the solution. Each measurement consisted of 5 runs and was repeated 5 times for each $\mathrm{pH}$ 
condition. Prior to the measurement, the system ran a number of background scans to eliminate any background noise that may affect the results.

A Malvern Mastersizer 2000 was used to determine the particle size distribution of gelatin microgels in a 10 micron filtered $\mathrm{pH}$ solution of 4, 7 and 10. Before measurement, a background measurement was performed to eliminate any contamination of background noise, dust on optics or contaminants floating within the system. The concentration of the gelatin microgels was of $3 \mathrm{~g} \mathrm{dm}^{-3}$. The agitation speed of $3000 \mathrm{rpm}$ was investigated, and each run consisted of 20 measurements. The process was repeated for each $\mathrm{pH}$ solution.

\section{Microscopic analysis}

The scanning electron microscope, Vega 3 Tescan, was used to examine surface morphology and coatings' thickness. The surface morphology was inspected at the top of the as-deposited coating. The actual coating thickness achieved was determined by examining the cross sections of nickel-plated copper samples following the BS EN ISO 9220:1995 standard as a guide. Energy dispersive X-ray microanalysis was employed to analyse the chemical composition of the coatings through elemental image mapping or spectrum.

A Zeiss - AX10 - Scope A1 fluorescent microscope with Zen Lite 2012 software was used to confirm the presence of microgels in Nilgelatin microgel composite coatings.

\section{Mechanical properties analysis}

Plint Tribology Products TE 66 Micro-Scale Abrasion Tester was used for wear tests. The tests were performed according to the BS EN ISO 2624:1016. Five samples of each coating and two control samples of polished, uncoated steel were used. The polished sides of the samples were tested. A force of $0.2 \mathrm{~N}$ was applied in all tests at $80 \mathrm{rpm}$. A weathered steel ball of diameter $25 \mathrm{~mm}$ was used for all tests. The samples were clamped in place so that no free movement can take place. An abrasive slurry was used between the ball and the sample 
surface, which was automatically fed through plastic tubing. All tests were non-perforating and consisted of 160, 200 and 400 revolutions.

The abrasive wear rate $\mathrm{K}_{\mathrm{c}}$ was calculated using the equation (1), where $\mathrm{R}$ is the radius of the ball; $\mathrm{b}$ is the crater diameter; $\mathrm{S}$ is the sliding distance and $\mathrm{N}$ is the normal load.

$$
\mathrm{K}_{\mathrm{c}}=\pi \times \mathrm{b}^{4} \backslash 64 \times \mathrm{R} \times \mathrm{S} \times \mathrm{N}
$$

The Vickers microhardness test was performed on a flat surface and a cross-section of coatings. The load of $10 \mathrm{~g}$ was used at dwelling time of 15 seconds. Three different samples of each coating type and an uncoated control sample were tested.

\section{Corrosion analysis}

The corrosion resistance of the coatings was evaluated by electrochemical impedance spectroscopy (EIS). Measurements were carried out in a $3.5 \%$ wt. $\mathrm{NaCl}$ solution using a Gamry ParaCell ${ }^{\mathrm{TM}}$ Electrochemical Cell Kit connected to a Solartron analytical potentiostat. A graphite plate was used as the counter electrode. $\mathrm{An} \mathrm{Ag} / \mathrm{AgCl}$ saturated reference electrode was employed. An area of $2.6 \mathrm{~cm}^{2}$ of the coated samples was exposed to the $\mathrm{NaCl}$ solution. The EIS measurements were taken after $0.5,2,5,11$, and 20 hours in the corrosive medium using Modulab XM software. The impedance measurements were conducted at corrosion potentials and sinusoidal voltage excitation with the amplitude of $10 \mathrm{mV}$ in a frequency range of $10 \mathrm{kHz}$ to $0.008 \mathrm{~Hz}$.

\section{Results and discussion}

\section{Microgels characterisation}

The zeta potential of gelatin microgels was investigated to understand the behaviour of the particles in the solution better and to aid in selecting bath's composition and parameters for electroplating. The more acidic solution resulted in the formation of a positive charge layer across the surface, compared to an alkaline solution that resulted in a negative charge. It is in 
agreement with the results described in [10]. Figure 1 shows the effect of $\mathrm{pH}$ on the zeta potential in de-ionised water. Clearly, as the $\mathrm{pH}$ of the solution increased the zeta decreased from $20 \mathrm{mV}$ at $\mathrm{pH} 4.1$ to $-20 \mathrm{mV}$ at $\mathrm{pH} 10$. This can be explained by the absorption of $\mathrm{H}+$ and $\mathrm{OH}$ - ions on gelatin microgels at lower and higher $\mathrm{pH}$ levels, respectively. The increase in $\mathrm{pH}$ results in gradually moving the zeta potential to zero. At the isoelectric point, the net charge on the surface is highly unstable, and flocculation was likely to occur. The isoelectric point for gelatin microgels felt between $\mathrm{pH} 8$ and 9. A similarly shaped correlation was observed for gelatin nanoparticles in a drug delivery study [11]. The low zeta potential values observed for gelatin microgels may aid in explaining the aggregation of observed within the bath due to the low repulsive forces exhibited by the particles, which then resulted in a poor dispersion throughout the coating.

Figure 2 shows the average particle size distribution for $\mathrm{pH}$ levels of 4,7 and 10 . Unexpectedly, the best particle size distribution was found to be achieved at $\mathrm{pH} 7$, close to the isoelectric point were particles exhibit no charge and are considered highly unstable due to the extremely low or neutral zeta potential. The electrostatic repulsion forces could have the minimal effect on the dispersion of particles within the solution since the zeta potential results showed shallow values below the stable region of $\pm 30 \mathrm{mV}$. Therefore, the dispersion stability of the particles was affected more by the concentration of the microgels within the solution. For example, a low concentration would result in an increased distance between particles at any given point, compared to a high concentration that results in particles in the very close proximity of one another increasing the chance of aggregation. Microgels swelling or the clustering of particles together within the bath resulting in larger particles being registered by the mastersizer may explain the larger particle sizes measured.

\section{Microscopic analysis of Nilgelatin microgels coating}


A successful co-deposition was achieved with goed distribution of the microgels distributed throughout the coating. However, areas of grouped microgels were also observed across the surface with sections of just nickel coating in between, figure $3 \mathrm{a}$. The grouping of microgels is likely due to aggregation within the bath during the plating process. The use of the EDX allowed a clear distinction to be made between the coating and the substrate material. On inspection, the coating appeared crack-free and homogenous with the thickness of around 10 $\mu \mathrm{m}$, figure $3 \mathrm{~b}$.

The fluorescent microscope was used to further confirm the incorporation of gelatin microgels in the nickel coating. Figure 4 presents the example image of the successful incorporation of gelatin microgels, highlighted as the white dots scattered across the coating surface.

\section{Mechanical properties of Ni and Ni|galetin microgels coatings}

The hardness test results are presented in Table 2. It can be seen that the microhardness of Nilgelatin microgels composite coatings was higher than that of pure Ni coating. The correlation between the strengthening mechanism of the second phase and the microhardness increment is still not well revealed. The reason for the increase in hardness could be the reduction of the grain size of the nickel matrix $[12,13]$. Specifically, Hall-Petch strengthening could contribute to an increase in microhardness of Nilgelatin microgels composite coatings [14]. In most cases, composite coatings based on metallic matrices have higher hardness than pure metal coatings because of finer-grained structure and dispersed particles obstructing the dislocation movement [12]. The growth of microhardness of composite nickel-based coatings was observed for hard and soft particles inclusion [14-16].

The results of the abrasive wear rates can be seen in Table 3. The abrasive rate of nickel coatings was lower than that of steel. Within the nickel coatings, Nilgelatin microgels ones exhibited better wear resistance. The total weight loss in the pure nickel coating is over one and a half times that in the Nilgelatin microgels composite coating. It seems that better wear 
resistance of Nilgelatin microgels composite coating compared to pure Ni coating is a direct consequence of the increase in surface hardness of the coatings. To conclude, the wear behaviour of all the coatings is in agreement with the general understanding that the harder coatings have the higher wear resistance.

\section{Corrosion resistance of $\mathrm{Ni}$ and Ni|gelatin microgels coatings}

Corrosion resistance of both types of coatings was assessed during 20 hours of immersion in the $3.5 \%$ wt. $\mathrm{NaCl}$ solution. The coatings exhibited different behaviour. Plain nickel coating showed typical active corrosion, without apparent diffusion limitations, as presented in the Nyquist plot, figure 5 a. The Nilgelatin microgels coating was characterised by two, poorly separated, time constants in the Nyquist plot, figure $5 \mathrm{~b}$. For fitting of experimental impedance spectra, two basic electrical equivalent circuits were employed, figure 6. First of them, comprised only the resistance of electrolytic solution $\left(\mathrm{R}_{\mathrm{s}}\right)$, charge transfer resistance $\left(\mathrm{R}_{\mathrm{ct}}\right)$ and a constant phase element $\left(\mathrm{CPE}_{\mathrm{dl}}\right)$ describing the double layer capacitance, figure $6 \mathrm{a}$. The second one consisted additionally of the resistance of the electrolytic solution in the surface layer of the coating $\left(\mathrm{R}_{\mathrm{coat}}\right)$ and the electric capacitance of this layer, represented by another constant phase element $\left(\mathrm{CPE}_{\text {coat }}\right)$, figure $6 \mathrm{~b}$.

It can be stated that the corrosion resistance of the nickel coating gradually decreased between 0.5 and 20 hours of the exposure to the corrosive medium. That was evidenced by a decrease of charge transfer resistance, from 28 to $14 \mathrm{k} \Omega \mathrm{cm}^{2}$, Fig. 7. The corrosion resistance of the Nilgelatin microgels coating was one order of magnitude lower at the beginning of the immersion in $\mathrm{NaCl}$ solution, comparing to the Nilgelatin microgels coating. However, the direction of changes in the $R_{c t}$ values was the opposite than in the case of the plain nickel coating. Between 0.5 and 20 hours of the exposure, $\mathrm{R}_{\mathrm{ct}}$ increased from 5.8 to $10 \mathrm{k} \Omega \mathrm{cm}^{2}$, figure 7. It is likely that due to the dissolution of gelatin microgels, thin layer acting as a physical barrier was built on the nickel surface. The evolution of the $\mathrm{R}_{\text {coat }}$ value supported this 
supposition. It also increased, from 570 to $910 \Omega \mathrm{cm}^{2}$, between 0.5 and 20 hours of the immersion, figure 7. The gelatin molecules can form a metal/gelatin complex on the metal surface utilising backbone amide linkage of the polypeptide chain [17]. Gelatin was also described as a mixed-type inhibitor of the mild steel corrosion in acidic media. It can block both the cathodic and anodic reaction sites [18].

This observation may lead to the conclusion that gelatin microgels met the expectations and did not accelerate the corrosion of nickel. Therefore, the microgels could be used as carriers of corrosion inhibitors.

\section{Conclusions}

The gelatin microgels are promising candidates for the nickel-based coating functionalisation and can be used as carriers for corrosion inhibitors.

It was demonstrated that the microgels could be co-deposited with the nickel matrix by the electroplating method. The microgels were detected in the coatings by scanning electron microscopy and fluorescence microscopy.

EIS measurements showed the beneficial effect of the gelatin microgels on the corrosion performance of the nickel-coated steel substrate, as the charge transfer resistance of the composite coatings increased with the immersion time in the corrosive medium.

The Nilgelatin microgels coatings exhibited enhanced hardness and wear resistance in comparison to the plain nickel coatings.

\section{Disclosure statement}

No potential conflict of interest was reported by the authors. 


\section{References}

[1] P. Sørensen, S. Kiil, K. Dam-Johansen, C. Weinell, Anticorrosive coatings: a review, J Coat Technol Res 6 (2009) 135-176. https://doi.org/10.1007/s11998-008-9144-2.

[2] A. Makhlouf, Handbook of smart coatings for materials protection, Woodhead Publishing, London, 2014.

[3] M. Samadzadeh, S. Boura, M. Peikari, S. Kasiriha, A. Ashrafi, A review on self-healing coatings based on micro/nanocapsules, Prog. Org. Coat. 68 (2010) 159-164.

https://doi.org/10.1016/j.porgcoat.2010.01.006.

[4] T. Nesterova, K. Dam-Johansen, S. Kiil, Synthesis of durable microcapsules for selfhealing anticorrosive coatings: A comparison of selected methods, Prog. Org. Coat. 70 (2011) 342-352. https://doi.org/10.1016/j.porgcoat.2010.09.032.

[5] Y. Zhao, W. Zhang, L. Liao, S. Wang, W. Li, Self-healing coatings containing microcapsule, Appl. Surf. Sci. 252 (2012) 1915-1918.

https://doi.org/10.1016/j.apsusc.2011.06.154.

[6] A. Latnikova, D. Grigoriev, M. Schenderlein, H. Möhwald, D. Shchukin, A new approach towards "active" self-healing coatings: exploitation of microgels, Soft Matter 8 (2012) https://doi.org/10.1039/C2SM26100F.

[7] A. Stankiewicz, Z. Jagoda, K. Zielińska, I. Szczygieł, Gelatin microgels as a potential corrosion inhibitor carriers for self-healing coatings: Preparation and codeposition, Mater. Corros. 66 (2015) 1391-1396. https://doi.org/10.1002/maco.201508436.

[8] J. Kelly, S. Goods, A. Talin, J. Hachman, Electrodeposition of Ni from Low-Temperature Sulfamate Electrolytes, J. Electrochem. Soc. 153 (2006) C318-C324.

https://doi.org/10.1149/1.2181439.

[9] D. Baudrand, Nickel sulfamate plating, its mystique and practicality, Metal Finishing 94 (1996) 15-18. https://doi.org/10.1016/0026-0576(96)81353-5.

[10] R. Schrieber, H. Gareis, Gelatine Handbook, Wiley, Weinheim, 2007.

[11] S. Azarmi, Y, Huang, H. Chen, S. McQuarrie, D. Abrams, W. Roa, W. H. Finlay, G. G. Miller, R. Löbenberg, Optimization of a two-step desolvation method for preparing gelatin nanoparticles and cell uptake studies in 143B osteosarcoma cancer cells, J Pharm Pharm Sci 9 (2006) 124-132. https://doi.org/10.7939/R3J96097M.

[12] H.Gül, F. Kuhȩ, S. Astan, A. Alp, H. Akbulut, Characteristies of eletro edepesited Ni$\mathrm{Al}_{2} \mathrm{O}_{3}$ nano-particle reinforced metal matrix composite (MMC) coatings, Wear 267 (2009) 976 990. https://doi.org/10.1016/j.wear.2008.12.022.

L. M. Muresan, Electrodeposited Zn-Nanoparticle Composite Coatings for Corrosion Protection of Steel, Handbook of Nanoelectrochemistry, Springer International Publishing Switzerland 2015. https://doi.org/10.1007/978-3-319-15207-3_34-1 
[13] N. Hansen, B. Ralph, Additive strengthening mechanisms in dispersion hardened polyerystals, Acta Metallurgica 34 (1986) 1955-1962. https://doi.org/10.1016/0001 6160(86)90254-3.

S. Sangeetha, G. Paruthimal Kalaignana, J. Tennis Anthuvan, Pulse electrodeposition of selflubricating Ni-W/PTFE nanocomposite coatings on mild steel surface, Applied Surface Science 359 (2015) 412-419. http://dx.doi.org/10.1016/j.apsusc.2015.10.127

[14] T. Borkar, S. Harimkar, Microstructure and wear behaviour of pulse electrodeposited NiCNT composite coatings, Surface Engineering 27 (2011) 524-530.

https://doi.org/10.1179/1743294410Y.0000000001.

[15] V. Medeliene, The influence of $\mathrm{B}_{4} \mathrm{C}$ and $\mathrm{SiC}$ additions on the morphological, physical, chemical and corrosion properties of Ni coatings, Surf. Coat. Technol. 154 (2002) 104-111. https://doi.org/10.1016/S0257-8972(01)01703-0.

[16] E. Pena-Munoz, P. Bercot, A. Grosjean, M. Rezrazi, J. Pagetti, Electrolytic and electroless coatings of Ni-PTFE composites Study of some characteristics, Surf. Coat. Technol. 107 (1998) 85-93. https://doi.org/10.1016/S0257-8972(98)00547-7.

[17] K. Haruna , I. B. Obot, N. K. Ankah, A. A. Sorour, T. A. Saleh, Gelatin: A green corrosion inhibitor for carbon steel in oil well acidizing environment, J. Mol. Liq. 264 (2018) 515-525. https://doi.org/10.1016/j.molliq.2018.05.058.

[18] A. Pal, S. Dey, D. Sukul. Effect of temperature on adsorption and corrosion inhibition characteristics of gelatin on mild steel in hydrochloric acid medium, Res. Chem. Intermed. 42 (2016) 4531-4549. https://doi.org/10.1007/s11164-015-2295-8. 


\section{Tables}

Table 1. Composition of the electroplating bath and electrodeposition parameters.

Table 2. Results of Vickers microhardness test.

Table 3. Average abrasive wear rates and material volume loss of coatings.

\section{Figure captions}

Figure 1. Dependence of zeta potential of gelatin microgels on $\mathrm{pH}$ of the solution.

Figure 2. Gelatin microgels size distribution at different $\mathrm{pHs}$.

Figure 3. SEM microphotographs of the Nilgelatin microgels coating: a) as-deposited surface;

b) cross section mapping (Cu-orange; Ni-yellow; C-blue; Si-green; O-red).

Figure 4. Fluorescence microscope photograph of the Nilgelatin microgels coating.

Figure 5. Nyquist plots of EIS data obtained from a) the nickel coating and b) the Nilgelatin microgels coating.

Figure 6. Models of equivalent circuits proposed for curve fitting of EIS data: a) the nickel coating and $b$ ) the Nilgelatin microgels coating.

Figure 7. Effect of the exposure time of nickel and Nilgelatin microgels coatings on the $\mathrm{R}_{\mathrm{ct}}$ and $R_{\text {coat }}$ values calculated from the data obtained from the fitting procedure of experimental impedance spectra. 

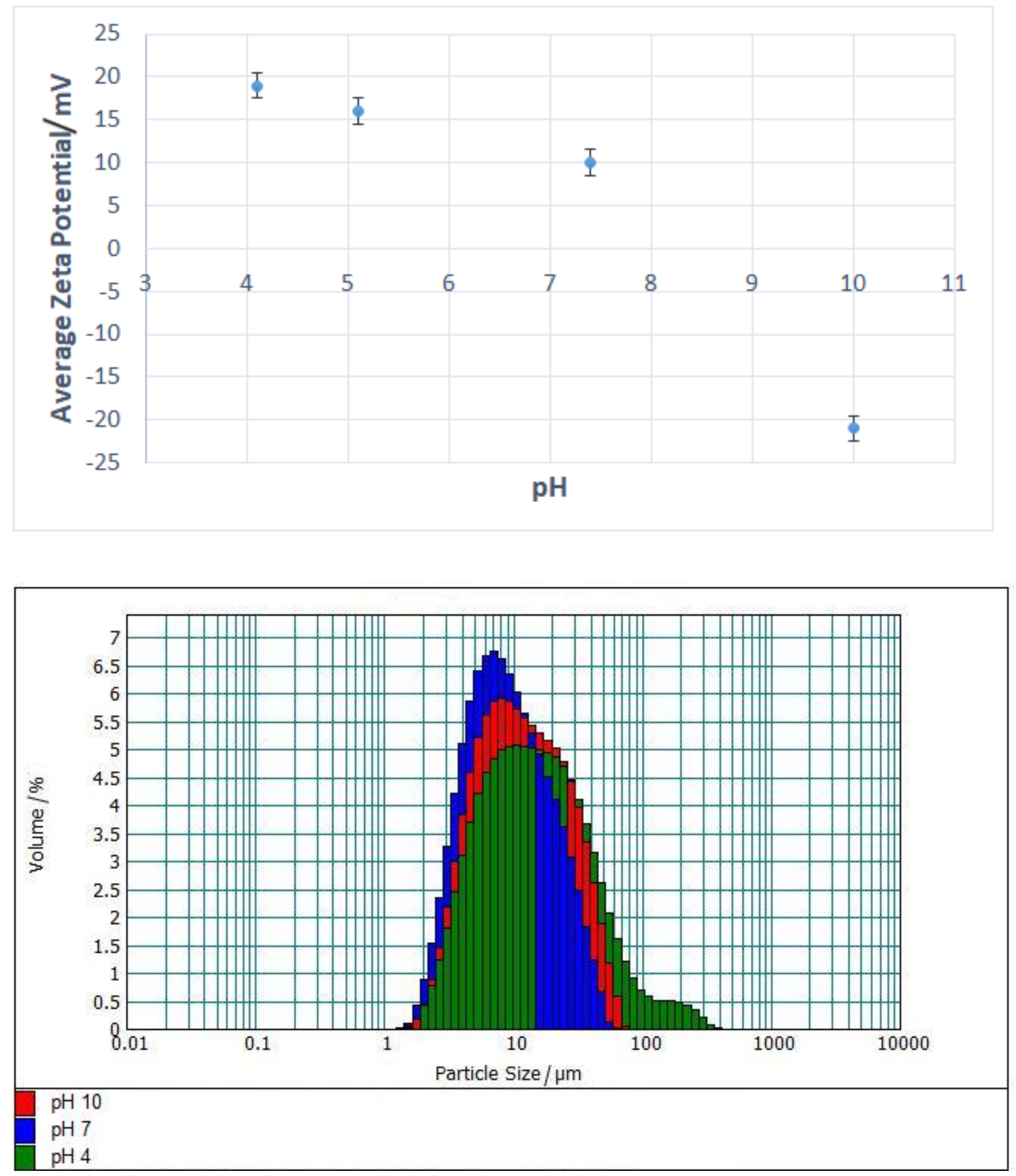

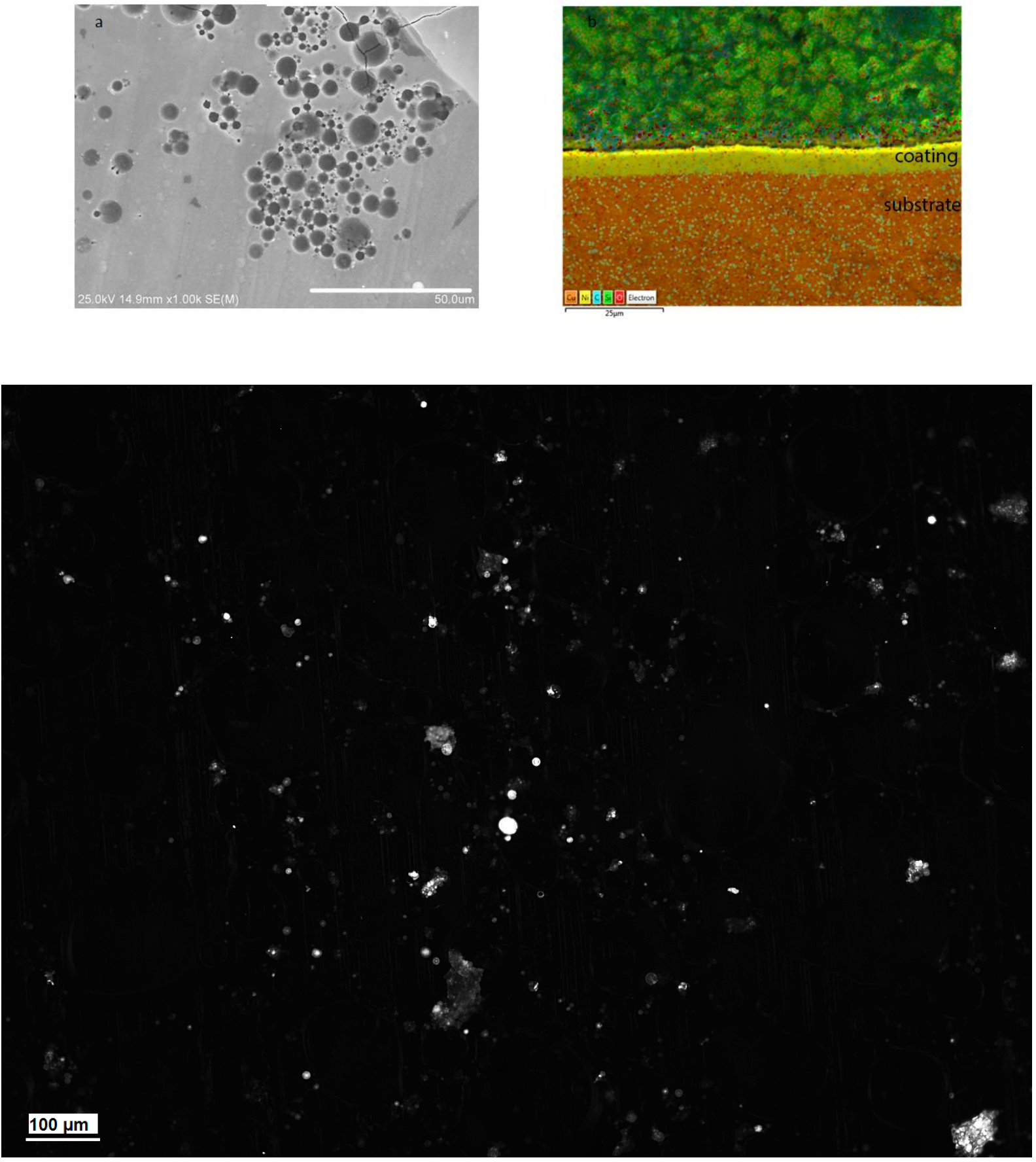

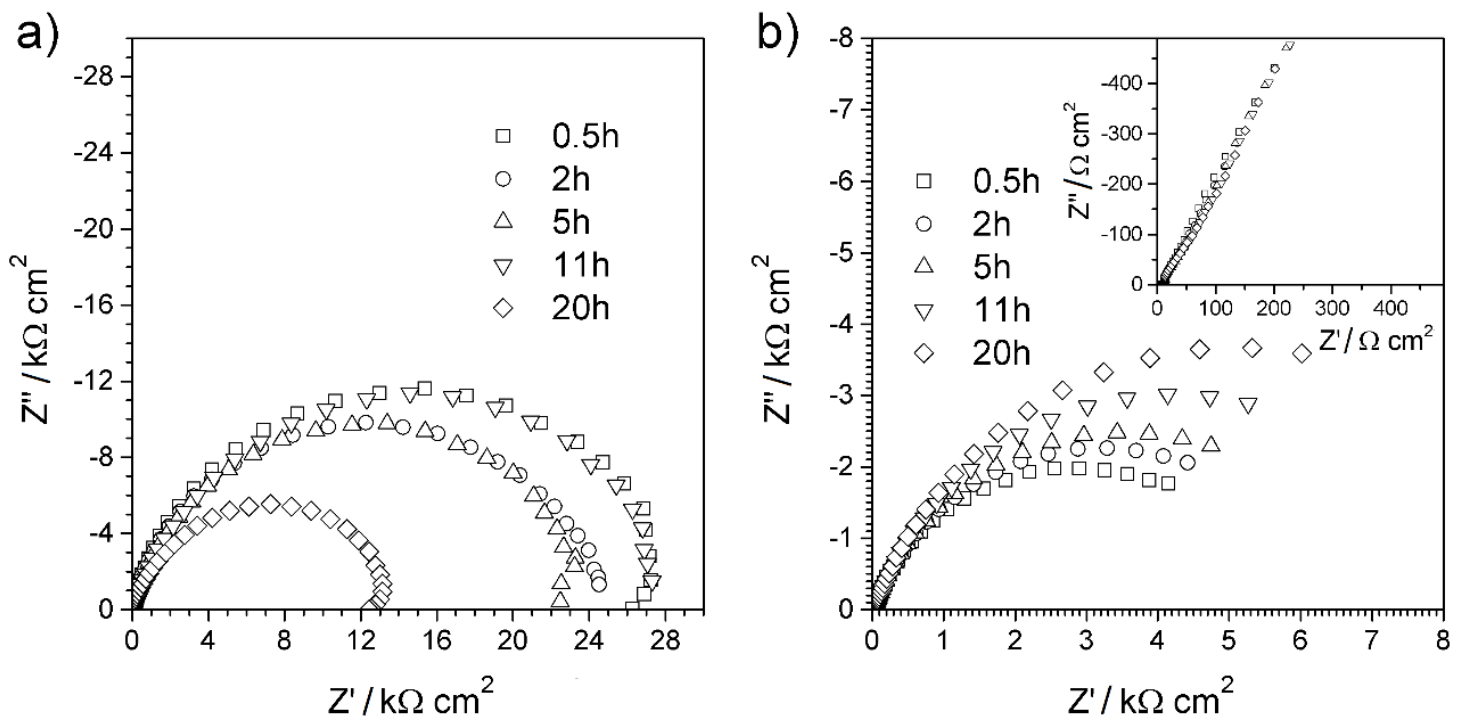

a)

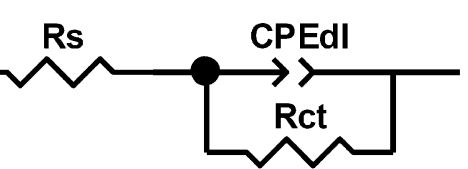

b)
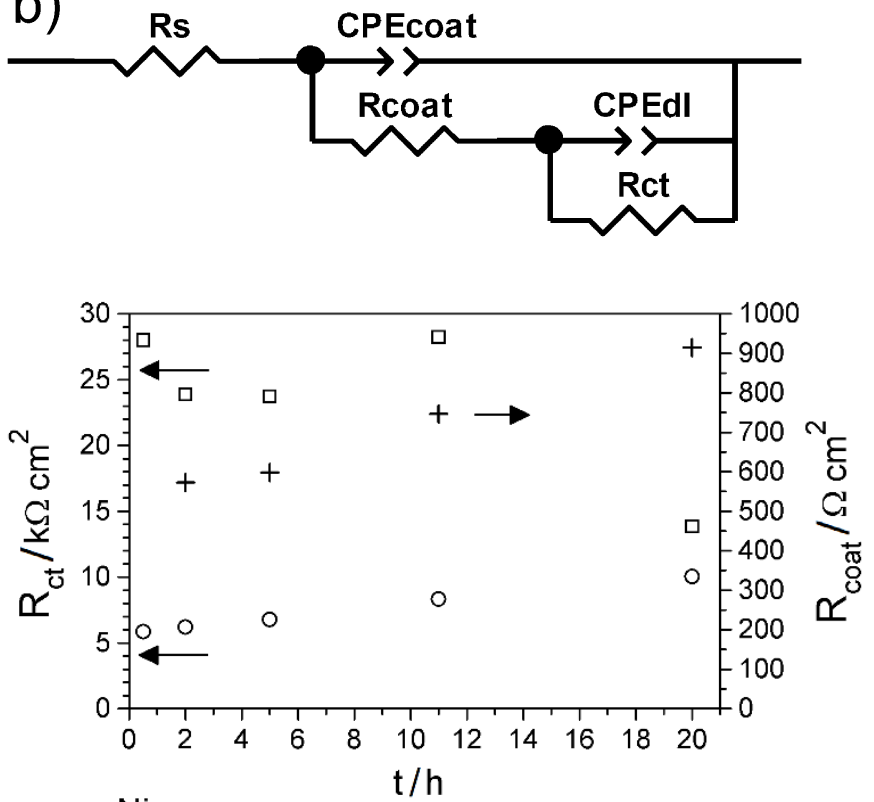

व $\mathrm{Ni}$

- Ni/microgels $+\mathrm{Ni} /$ microgels 